\title{
Asymptotics of Solutions for fully Nonlinear Elliptic Problems at Nearly Critical Growth
}

\author{
A. Musesti and M. Squassina
}

\begin{abstract}
In this paper we deal with the study of limits of solutions of a class of fully nonlinear elliptic problems at nearly critical growth. By means of P.L. Lions' concentration-compactness principle, we prove an alternative result for the existence of non-trivial solutions of the limit problem.
\end{abstract}

Keywords: Concentration-compactness principle, critical exponent, best Sobolev constant, fully nonlinear elliptic problems

AMS subject classification: $35 \mathrm{~J} 65,35 \mathrm{~B} 40$

\section{Introduction}

Let $\Omega$ be a bounded domain of $\mathbb{R}^{n}, 1<p<n$ and $p^{*}=\frac{n p}{n-p}$. In 1989 Guedda and Veron [10] proved that the $p$-Laplacian problem at critical growth

$$
\left.\begin{array}{rlrl}
-\Delta_{p} u & =u^{p^{*}-1} & & \text { in } \Omega \\
u>0 & & \text { in } \Omega \\
u & =0 & & \text { on } \partial \Omega
\end{array}\right\}
$$

has no non-trivial solution $u \in W_{0}^{1, p}(\Omega)$ if the domain $\Omega$ is star-shaped. As known, this non-existence result is due to the failure of compactness for the critical Sobolev embedding $W_{0}^{1, p}(\Omega) \hookrightarrow L^{p^{*}}(\Omega)$, which causes a loss of global Palais-Smale condition for the functional associated with problem $(*)$. On the other hand, if for instance one considers annular domains

$$
\Omega_{r_{1}, r_{2}}=\left\{x \in \mathbb{R}^{n}: 0<r_{1}<|x|<r_{2}\right\}
$$

Both authors: Dip. di Matem. e Fisica, Via Musei 41, I-25121 Brescia, Italy squassin@dmf.unicatt.it and http://www.dmf.unicatt.it/ squassin musesti@dmf.unicatt.it and http://www.dmf.unicatt.it/ musesti 
then the radial embedding

$$
W_{0, \text { rad }}^{1, p}\left(\Omega_{r_{1}, r_{2}}\right) \hookrightarrow L^{q}\left(\Omega_{r_{1}, r_{2}}\right)
$$

is compact for each $q<+\infty$ and one can find a non-trivial radial solution of problem $(*)$ (see [11]). In particular, the existence of non-trivial solutions of problem $(*)$ depends also on the topology of the domain. In the case $p=2$, the problem

$$
\left.\begin{array}{rlrl}
-\Delta u & =u^{(n+2) /(n-2)} & & \text { in } \Omega \\
u & >0 & & \text { in } \Omega \\
u & =0 & & \text { on } \partial \Omega
\end{array}\right\}
$$

has been deeply studied and existence results have been obtained provided that $\Omega$ satisfies suitable assumptions. In the striking paper [3], Bahri and Coron have proved that if $\Omega$ has a non-trivial topology, i.e. if $\Omega$ has a nontrivial homology in some positive dimension, then problem $(* *)$ always admits a non-trivial solution.

On the other hand, Dancer [8] constructed for each $n \geq 3$ a contractible domain $\Omega_{n}$, homeomorphic to a ball, for which problem (**) has a non-trivial solution. Therefore, we see how the existence of non-trivial solutions of problem $(* *)$ is related to the shape of the domain and not just to the topology. See also [15] and references therein for more recent existence and multiplicity results.

We remark that, to the authors' knowledge, this kind of achievements are not known when $p \neq 2$. In our opinion, one of the main difficulties is the fact that, differently from the case $p=2$, it is not proven that all positive smooth solutions of the equation $-\Delta_{p} u=u^{p^{*}-1}$ in $\mathbb{R}^{n}$ are Talenti's radial functions, which attain the best Sobolev constant (see Proposition 3.1).

Now, there is a second approach in the study of problem $(*)$, which in general does not require any geometrical or topological assumption on $\Omega$, namely to investigate the asymptotic behaviour of solutions $u_{\varepsilon}$ of problems with nearly critical growth

$$
\left.\begin{array}{rlrl}
-\Delta_{p} u & =|u|^{p^{*}-2-\varepsilon} u & & \text { in } \Omega \\
u & =0 & & \text { on } \partial \Omega
\end{array}\right\}
$$

as $\varepsilon \rightarrow 0$. If $\Omega$ is a ball and $p=2$, Atkinson and Peletier [2] showed in 1987 the blow-up of a sequence of radial solutions. The extension to the case $p \neq 2$ was achieved by Knaap and Peletier [12] in 1989. On a general bounded domain, instead, the study of limits of solutions of problem $(* * *)$ was performed by Garcia Azorero and Peral Alonso [9] around 1992. 
Let now $\varepsilon>0$ and consider the general class of Euler-Lagrange equations with nearly critical growth

$$
\left.\begin{array}{rlrl}
-\operatorname{div}\left(\nabla_{\xi} \mathcal{L}(x, u, \nabla u)\right)+D_{s} \mathcal{L}(x, u, \nabla u) & =|u|^{p^{*}-2-\varepsilon} u & & \text { in } \Omega \\
u & =0 & & \text { on } \partial \Omega
\end{array}\right\}
$$

associated with the functional $f_{\varepsilon}: W_{0}^{1, p}(\Omega) \rightarrow \mathbb{R}$ given by

$$
f_{\varepsilon}(u)=\int_{\Omega} \mathcal{L}(x, u, \nabla u) d x-\frac{1}{p^{*}-\varepsilon} \int_{\Omega}|u|^{p^{*}-\varepsilon} d x .
$$

As noted in [18], in general these functionals are not even locally Lipschitz under natural growth assumptions. Nevertheless, via techniques of non-smooth critical point theory (see [18] and references therein) it can be shown that for each $\varepsilon>0$ problem $\left(\mathcal{P}_{\varepsilon}\right)$ admits a non-trivial solution $u_{\varepsilon} \in W_{0}^{1, p}(\Omega)$.

Let $u_{\varepsilon}$ be a solution of problem $\left(\mathcal{P}_{\varepsilon}\right)$. The main goal of this paper is to prove that if the weak limit of $\left(\left|\nabla u_{\varepsilon}\right|^{p}\right)_{\varepsilon>0}$ has no blow-up points in $\Omega$, then the limit problem

$$
\left.\begin{array}{rlrl}
-\operatorname{div}\left(\nabla_{\xi} \mathcal{L}(x, u, \nabla u)\right)+D_{s} \mathcal{L}(x, u, \nabla u) & =|u|^{p^{*}-2} u & & \text { in } \Omega \\
u & =0 & & \text { on } \partial \Omega
\end{array}\right\}
$$

has a non-trivial solution (the weak limit of $\left(u_{\varepsilon}\right)_{\varepsilon>0}$ ), provided that $f_{\varepsilon}\left(u_{\varepsilon}\right) \rightarrow c$ with

$$
\frac{p^{*}-p-\gamma}{p p^{*}}(\nu S)^{\frac{n}{p}}<c<2 \frac{p^{*}-p-\gamma}{p p^{*}}(\nu S)^{\frac{n}{p}}
$$

where $\nu>0$ and $\gamma \in\left(0, p^{*}-p\right)$ will be introduced later on. In our framework, (2) plays the role of a generalized second critical energy range (if $\gamma=0$ and $\nu=1$, one finds the usual range $\frac{S^{n / p}}{n}<c<2 \frac{S^{n / p}}{n}$ for problem $\left.(* * *)\right)$.

The plan of the paper is as follows:

In Section 2 we shall state our main results. Section 3 contains some preliminary lemmas, namely the lower bounds of the non-vanishing Dirac masses and of the non-trivial weak limits. In Section 4 we prove our main results. In Section 5 we see that at the mountain pass levels the sequence $\left(u_{\varepsilon}\right)_{\varepsilon>0}$ blows up. Finally, Section 6 contains a non-existence result. 


\section{The main results}

Let $\Omega$ be any bounded domain of $\mathbb{R}^{n}$. In the following, the space $W_{0}^{1, p}(\Omega)$ will be endowed with the standard norm $\|u\|_{1, p}^{p}=\int_{\Omega}|\nabla u|^{p} d x$ and $\|\cdot\|_{p}$ will denote the usual norm of $L^{p}(\Omega)$.

Assume that $\mathcal{L}: \Omega \times \mathbb{R} \times \mathbb{R}^{n} \rightarrow \mathbb{R}$ is measurable in $x$ for all $(s, \xi) \in \mathbb{R} \times \mathbb{R}^{n}$, of class $C^{1}$ in $(s, \xi)$ a.e. in $\Omega$, that $\mathcal{L}(x, s, \cdot)$ is strictly convex and $\mathcal{L}(x, s, 0)=0$. Moreover, assume the following:

$\left(\mathcal{A}_{1}\right)$ There exists $b_{0}>0$ such that

$$
\mathcal{L}(x, s, \xi) \leq b_{0}|s|^{p}+b_{0}|\xi|^{p}
$$

for a.e. $x \in \Omega$ and for all $(s, \xi) \in \mathbb{R} \times \mathbb{R}^{n}$.

$\left(\mathcal{A}_{2}\right)$ There exists $b_{1}>0$ such that for each $\delta>0$ there exists $a_{\delta} \in L^{1}(\Omega)$ with

$$
\left|D_{s} \mathcal{L}(x, s, \xi)\right| \leq a_{\delta}(x)+\delta|s|^{p^{*}}+b_{1}|\xi|^{p}
$$

for a.e. $x \in \Omega$ and for all $(s, \xi) \in \mathbb{R} \times \mathbb{R}^{n}$. Moreover, there exist $a_{1} \in L^{p^{\prime}}(\Omega)$ and $\nu>0$ such that

$$
\begin{gathered}
\left|\nabla_{\xi} \mathcal{L}(x, s, \xi)\right| \leq a_{1}(x)+b_{1}|s|^{\frac{p^{*}}{p^{\prime}}}+b_{1}|\xi|^{p-1}, \\
\nabla_{\xi} \mathcal{L}(x, s, \xi) \cdot \xi \geq \nu|\xi|^{p}
\end{gathered}
$$

for a.e. $x \in \Omega$ and for all $(s, \xi) \in \mathbb{R} \times \mathbb{R}^{n}$.

$\left(\mathcal{A}_{3}\right)$ For a.e. $x \in \Omega$ and for all $(s, \xi) \in \mathbb{R} \times \mathbb{R}^{n}$,

$$
D_{s} \mathcal{L}(x, s, \xi) s \geq 0
$$

and there exists $\gamma \in\left(0, p^{*}-p\right)$ such that

$$
(\gamma+p) \mathcal{L}(x, s, \xi)-\nabla_{\xi} \mathcal{L}(x, s, \xi) \cdot \xi-D_{s} \mathcal{L}(x, s, \xi) s \geq 0
$$

for a.e. $x \in \Omega$ and for all $(s, \xi) \in \mathbb{R} \times \mathbb{R}^{n}$.

Remark 2.1. The growth conditions of $\left(\mathcal{A}_{1}\right)$ and $\left(\mathcal{A}_{2}\right)$ and the assumptions in $\left(\mathcal{A}_{3}\right)$ are natural in the fully nonlinear setting and were considered in [18], and in a stronger form in [1, 16] (see also Remark 6.2). Notice that when $\mathcal{L}$ is $p$-homogeneous with respect to $\xi$, then condition (8) becomes $D_{s} \mathcal{L}(x, s, \xi) s \leq \gamma \mathcal{L}(x, s, \xi)$ for a.e. $x \in \Omega$ and for all $(s, \xi) \in \mathbb{R} \times \mathbb{R}^{n}$.

As an example, taking $A \in C^{1}(\mathbb{R}) \cap L^{\infty}(\mathbb{R})$ with $A^{\prime} \in L^{\infty}(\mathbb{R}), A(s) \geq \nu$ and $\gamma A(s) \geq A^{\prime}(s) s \geq 0$ for each $s \in \mathbb{R}$, the class of Lagrangians

$$
\mathcal{L}(x, s, \xi)=\frac{1}{p} A(s)|\xi|^{p}
$$


satisfies all the previous requirements. For instance, $\left(\gamma^{-1}+\arctan \left(s^{2}\right)\right)|\xi|^{p} / p$ belongs to this class for each $\gamma \in\left(0, p^{*}-p\right)$.

Remark 2.2. We stress that although as noted in the introduction $f_{\varepsilon}$ fails to be differentiable, one may compute the derivatives along the $L^{\infty}$-directions, namely

$f_{\varepsilon}^{\prime}(u)(\varphi)=\int_{\Omega} \nabla_{\xi} \mathcal{L}(x, u, \nabla u) \cdot \nabla \varphi d x+\int_{\Omega} D_{s} \mathcal{L}(x, u, \nabla u) \varphi d x-\int_{\Omega}|u|^{p^{*}-2-\varepsilon} u \varphi d x$

for all $u \in W_{0}^{1, p}(\Omega)$ and for all $\varphi \in W_{0}^{1, p} \cap L^{\infty}(\Omega)$.

The following is a general property due to Brézis and Browder [5].

Proposition 2.3. Let $u, v \in W_{0}^{1, p}(\Omega)$ be such that $D_{s} \mathcal{L}(x, u, \nabla u) v \geq 0$ and

$$
\langle w, \varphi\rangle=\int_{\Omega} \nabla_{\xi} \mathcal{L}(x, u, \nabla u) \cdot \nabla \varphi d x+\int_{\Omega} D_{s} \mathcal{L}(x, u, \nabla u) \varphi d x \quad\left(\varphi \in C_{c}^{\infty}(\Omega)\right)
$$

with $w \in L_{\text {loc }}^{1}(\Omega) \cap W^{-1, p^{\prime}}(\Omega)$. Then $D_{s} \mathcal{L}(x, u, \nabla u) v \in L^{1}(\Omega)$ and

$$
\langle w, v\rangle=\int_{\Omega} \nabla_{\xi} \mathcal{L}(x, u, \nabla u) \cdot \nabla v d x+\int_{\Omega} D_{s} \mathcal{L}(x, u, \nabla u) v d x .
$$

From now on, by solution of problem $\left(\mathcal{P}_{\varepsilon}\right)$ we shall always mean weak solution, namely $f_{\varepsilon}^{\prime}\left(u_{\varepsilon}\right)=0$ in the sense of distributions. The next lemma is our starting point.

Lemma 2.4. For each $\varepsilon>0,\left(\mathcal{P}_{\varepsilon}\right)$ admits a non-trivial solution $u_{\varepsilon} \in$ $W_{0}^{1, p}(\Omega)$.

\section{Proof. See [18: Theorem 1.1]}

We point out that, in our general framework, the technical aspects in the verification of the Palais-Smale condition for $f_{\varepsilon}$ are, in our opinion, interesting and not trivial.

Note that since $\mathcal{L}(x, s, 0)=0$, in view of (6) one obtains

$$
\mathcal{L}(x, s, \xi) \geq \frac{\nu}{p}|\xi|^{p}
$$

for a.e. $x \in \Omega$ and for all $(s, \xi) \in \mathbb{R} \times \mathbb{R}^{n}$.

Lemma 2.5. Let $\left(u_{\varepsilon}\right)_{\varepsilon>0} \subset W_{0}^{1, p}(\Omega)$ be a sequence of solutions of problem $\left(\mathcal{P}_{\varepsilon}\right)$ such that $\lim _{\varepsilon \rightarrow 0} f_{\varepsilon}\left(u_{\varepsilon}\right)<+\infty$. Then $\left(u_{\varepsilon}\right)_{\varepsilon>0}$ is bounded in $W_{0}^{1, p}(\Omega)$. 
Proof. For each $\varepsilon>0$ we have $f_{\varepsilon}^{\prime}\left(u_{\varepsilon}\right)(\varphi)=0$ for each $\varphi \in C_{c}^{\infty}(\Omega)$. On the other hand, taking into account $(7)$, by Proposition 2.3 one can also take $\varphi=u_{\varepsilon}$. Therefore, in view of (8) and (9) one obtains

$$
\begin{aligned}
\lim _{\varepsilon \rightarrow 0} f_{\varepsilon}\left(u_{\varepsilon}\right)= & \lim _{\varepsilon \rightarrow 0}\left(f_{\varepsilon}\left(u_{\varepsilon}\right)-\frac{1}{p^{*}-\varepsilon} f_{\varepsilon}^{\prime}\left(u_{\varepsilon}\right)\left(u_{\varepsilon}\right)\right) \\
= & \lim _{\varepsilon \rightarrow 0}\left(\int_{\Omega} \mathcal{L}\left(x, u_{\varepsilon}, \nabla u_{\varepsilon}\right) d x\right. \\
& -\frac{1}{p^{*}-\varepsilon} \int_{\Omega} \nabla_{\xi} \mathcal{L}\left(x, u_{\varepsilon}, \nabla u_{\varepsilon}\right) \cdot \nabla u_{\varepsilon} d x \\
& \left.-\frac{1}{p^{*}-\varepsilon} \int_{\Omega} D_{s} \mathcal{L}\left(x, u_{\varepsilon}, \nabla u_{\varepsilon}\right) u_{\varepsilon} d x\right) \\
\geq & \lim _{\varepsilon \rightarrow 0} \frac{p^{*}-p-\varepsilon-\gamma}{p^{*}-\varepsilon} \int_{\Omega} \mathcal{L}\left(x, u_{\varepsilon}, \nabla u_{\varepsilon}\right) d x \\
\geq & \frac{p^{*}-p-\gamma}{p p^{*}} \nu \lim _{\varepsilon \rightarrow 0} \int_{\Omega}\left|\nabla u_{\varepsilon}\right|^{p} d x .
\end{aligned}
$$

In particular, $\left(u_{\varepsilon}\right)_{\varepsilon>0}$ is bounded in $W_{0}^{1, p}(\Omega)$

Let us now recall the classical P.L. Lions' concentration-compactness principle

Lemma 2.6. Let $\left(u_{\varepsilon}\right)_{\varepsilon>0} \subset W_{0}^{1, p}(\Omega)$ be bounded and let $u$ be its weak limit. Then there exist two bounded positive measures $\mu$ and $\sigma$ such that

$$
\begin{aligned}
& \left|\nabla u_{\varepsilon}\right|^{p} \rightarrow \mu,\left|u_{\varepsilon}\right|^{p^{*}} \rightarrow \sigma \quad(\text { in the sense of measures }) \\
& \mu \geq|\nabla u|^{p}+\sum_{j=1}^{\infty} \mu_{j} \delta_{x_{j}} \quad\left(\mu_{j} \geq 0\right) \\
& \sigma=|u|^{p^{*}}+\sum_{j=1}^{\infty} \sigma_{j} \delta_{x_{j}} \quad\left(\sigma_{j} \geq 0\right) \\
& \mu_{j} \geq S \sigma_{j}^{\frac{p}{p^{*}}}
\end{aligned}
$$

where $\delta_{x_{j}}$ denotes the Dirac measure at $x_{j} \in \bar{\Omega}$ and $S$ denotes the best Sobolev constant for the embedding $W_{0}^{1, p}(\Omega) \hookrightarrow L^{p^{*}}(\Omega)$.

Proof. See, e.g., [13: Lemma I.1] or [14]

Under assumptions $\left(\mathcal{A}_{1}\right)-\left(\mathcal{A}_{3}\right)$, the following is our main result.

Theorem 2.7. Assume that $\left(u_{\varepsilon}\right)_{\varepsilon>0} \subset W_{0}^{1, p}(\Omega)$ is a sequence of solutions of problem $\left(\mathcal{P}_{\varepsilon}\right)$ such that $f_{\varepsilon}\left(u_{\varepsilon}\right) \rightarrow c$ and

$$
\frac{p^{*}-p-\gamma}{p p^{*}}(\nu S)^{\frac{n}{p}}<c<2 \frac{p^{*}-p-\gamma}{p p^{*}}(\nu S)^{\frac{n}{p}} .
$$


Then $\mu_{j}=0$ for $j \geq 2$ and the following alternative holds:

(a) $\mu_{1}=0$ and $u$ is a non-trivial solution of problem $\left(\mathcal{P}_{0}\right)$.

(b) $\mu_{1} \neq 0$ and $u=0$.

This result extends [9: Theorem 9] to a class of fully nonlinear elliptic problems.

Theorem 2.8. Let $\left(u_{\varepsilon}\right)_{\varepsilon>0}$ be any sequence of solutions of problem $\left(\mathcal{P}_{\varepsilon}\right)$ with

$$
\lim _{\varepsilon \rightarrow 0} f_{\varepsilon}\left(u_{\varepsilon}\right)=\frac{p^{*}-p-\gamma}{p p^{*}}(\nu S)^{\frac{n}{p}} .
$$

Then $u=0$.

As we shall see in Section 5, this is also the behaviour when one considers critical levels of mountain-pass type.

\section{The weak limit of $\left(u_{\varepsilon}\right)_{\varepsilon>0}$}

Let us briefly summarize the main properties of the best Sobolev constant [19].

Proposition 3.1. Let $1<p<n$ and $S$ be the best Sobolev constant, i.e.

$$
S=\inf \left\{\int_{\Omega}|\nabla u|^{p} d x: u \in W_{0}^{1, p}(\Omega) \text { with } \int_{\Omega}|u|^{p^{*}} d x=1\right\} .
$$

Then the following facts hold:

(a) $S$ is independent on $\Omega \subset \mathbb{R}^{n}$.

(b) The infimum (14) is never achieved on bounded domains $\Omega \subset \mathbb{R}^{n}$. $\mathbb{R}^{n}$

(c) The infimum (14) is achieved if $\Omega=\mathbb{R}^{n}$ by the family of functions on

$$
T_{\delta, x_{0}}(x)=\left(n \delta\left(\frac{n-p}{p-1}\right)^{p-1}\right)^{\frac{n-p}{p^{2}}}\left(\delta+\left|x-x_{0}\right|^{\frac{p}{p-1}}\right)^{-\frac{n-p}{p}}
$$

with $\delta>0$ and $x_{0} \in \mathbb{R}^{n}$. Moreover, $T_{\delta, x_{0}}$ is a solution of $-\Delta_{p} u=u^{p^{*}-1}$ on $\mathbb{R}^{n}$.

The next result establishes uniform lower bounds for the Dirac masses.

Lemma 3.2. If $\mu_{j} \neq 0$, then $\sigma_{j} \geq \nu^{\frac{n}{p}} S^{\frac{n}{p}}$ and $\mu_{j} \geq \nu^{\frac{n}{p^{*}}} S^{\frac{n}{p}}$.

Proof. Let $x_{j} \in \bar{\Omega}$ the point which supports the Dirac measure of coefficient $\sigma_{j}$. Denoting with $B\left(x_{j}, \delta\right)$ the open ball of center $x_{j}$ and radius $\delta>0$, we can consider a function $\psi_{\delta} \in C_{c}^{\infty}\left(\mathbb{R}^{n}\right)$ such that $0 \leq \psi_{\delta} \leq 1,\left|\nabla \psi_{\delta}\right| \leq \frac{2}{\delta}$, 
$\psi_{\delta}(x)=1$ if $x \in B\left(x_{j}, \delta\right)$ and $\psi_{\delta}(x)=0$ if $x \notin B\left(x_{j}, 2 \delta\right)$. By Proposition 2.3 we have

$$
\begin{aligned}
0= & f_{\varepsilon}^{\prime}\left(u_{\varepsilon}\right)\left(\psi_{\delta} u_{\varepsilon}\right) \\
= & \int_{\Omega} u_{\varepsilon} \nabla_{\xi} \mathcal{L}\left(x, u_{\varepsilon}, \nabla u_{\varepsilon}\right) \cdot \nabla \psi_{\delta} d x+\int_{\Omega} \psi_{\delta} \nabla_{\xi} \mathcal{L}\left(x, u_{\varepsilon}, \nabla u_{\varepsilon}\right) \cdot \nabla u_{\varepsilon} d x \\
& +\int_{\Omega} \psi_{\delta} D_{s} \mathcal{L}\left(x, u_{\varepsilon}, \nabla u_{\varepsilon}\right) u_{\varepsilon} d x-\int_{\Omega}\left|u_{\varepsilon}\right|^{p^{*}-\varepsilon} \psi_{\delta} d x
\end{aligned}
$$

Applying Hölder inequality and (5) to the first term of the decomposition and keeping into account that $\left(u_{\varepsilon}\right)_{\varepsilon>0}$ is bounded in $W_{0}^{1, p}(\Omega)$, one finds constants $c_{1}, c_{2}>0$ such that

$$
\begin{aligned}
\lim _{\varepsilon \rightarrow 0} \mid & \int_{\Omega} u_{\varepsilon} \nabla_{\xi} \mathcal{L}\left(x, u_{\varepsilon}, \nabla u_{\varepsilon}\right) \cdot \nabla \psi_{\delta} d x \mid \\
\leq & \left(\int_{B\left(x_{j}, 2 \delta\right)}\left|a_{1}\right|^{\frac{p}{p-1}} d x\right)^{\frac{p-1}{p}}\left(\int_{B\left(x_{j}, 2 \delta\right)}|u|^{p^{*}} d x\right)^{\frac{1}{p^{*}}}\left(\int_{B\left(x_{j}, 2 \delta\right)}\left|\nabla \psi_{\delta}\right|^{n} d x\right)^{\frac{1}{n}} \\
& +b_{1}\left(\int_{B\left(x_{j}, 2 \delta\right)}|u|^{p^{*}} d x\right)^{\frac{n-1}{n}}\left(\int_{B\left(x_{j}, 2 \delta\right)}\left|\nabla \psi_{\delta}\right|^{n} d x\right)^{\frac{1}{n}} \\
& +\widetilde{b}_{1}\left(\int_{B\left(x_{j}, 2 \delta\right)}|u|^{p^{*}} d x\right)^{\frac{1}{p^{*}}}\left(\int_{B\left(x_{j}, 2 \delta\right)}\left|\nabla \psi_{\delta}\right|^{n} d x\right)^{\frac{1}{n}} \\
\leq & c_{1}\left(\int_{B\left(x_{j}, 2 \delta\right)}|u|^{p^{*}} d x\right)^{\frac{1}{p^{*}}}+c_{2}\left(\int_{B\left(x_{j}, 2 \delta\right)}|u|^{p^{*}} d x\right)^{\frac{n-1}{n}} \\
= & \beta_{\delta}
\end{aligned}
$$

with $\beta_{\delta} \rightarrow 0$ as $\delta \rightarrow 0$. Then, taking into account (6) and (7) one has

$$
\begin{aligned}
0 & \geq-\beta_{\delta}+\lim _{\varepsilon \rightarrow 0} \nu \int_{\Omega}\left|\nabla u_{\varepsilon}\right|^{p} \psi_{\delta} d x-\lim _{\varepsilon \rightarrow 0} \mathcal{L}^{n}(\Omega)^{\frac{\varepsilon}{p^{*}}}\left(\int_{\Omega}\left|u_{\varepsilon}\right|^{p^{*}} \psi_{\delta} d x\right)^{\frac{p^{*}-\varepsilon}{p^{*}}} \\
& \geq-\beta_{\delta}+\nu \int_{\Omega} \psi_{\delta} d \mu-\int_{\Omega} \psi_{\delta} d \sigma
\end{aligned}
$$

Letting $\delta \rightarrow 0$, it results $\nu \mu_{j} \leq \sigma_{j}$. By means of (13) the proof is complete

In the next result we obtain uniform lower bounds for the non-zero weak limits.

Lemma 3.3. If $u \neq 0$, then $\int_{\Omega}|\nabla u|^{p} d x>\nu^{\frac{n}{p^{*}}} S^{\frac{n}{p}}$ and $\int_{\Omega}|u|^{p^{*}} d x>$ $\nu^{\frac{n}{p}} S^{\frac{n}{p}}$. 
Proof. By Lemma 3.2 we may assume that $\mu$ has at most $r$ Dirac masses $\mu_{1}, \ldots, \mu_{r}$ at $x_{1}, \ldots, x_{r}$, respectively. Let now $0<\delta<\frac{1}{4} \min \left\{\left|x_{i}-x_{j}\right|: i \neq j\right\}$ and $\psi_{\delta} \in C_{c}^{\infty}\left(\mathbb{R}^{n}\right)$ be such that $0 \leq \psi_{\delta} \leq 1,\left|\nabla \psi_{\delta}\right| \leq \frac{2}{\delta}, \psi_{\delta}(x)=1$ if $x \in B\left(x_{j}, \delta\right)$ and $\psi_{\delta}(x)=0$ if $x \notin B\left(x_{j}, 2 \delta\right)$. Taking into account (7), for each $\varepsilon, \delta>0$ we have

$$
\int_{\Omega} D_{s} \mathcal{L}\left(x, u_{\varepsilon}, \nabla u_{\varepsilon}\right) u_{\varepsilon}\left(1-\psi_{\delta}\right) d x \geq 0
$$

Then, since one can choose $\left(1-\psi_{\delta}\right) u_{\varepsilon}$ as test, by (6) one obtains

$$
\begin{aligned}
0= & f_{\varepsilon}^{\prime}\left(u_{\varepsilon}\right)\left(\left(1-\psi_{\delta}\right) u_{\varepsilon}\right) \\
= & \int_{\Omega} \nabla_{\xi} \mathcal{L}\left(x, u_{\varepsilon}, \nabla u_{\varepsilon}\right) \cdot \nabla u_{\varepsilon}\left(1-\psi_{\delta}\right) d x \\
& -\int_{\Omega} u_{\varepsilon} \nabla_{\xi} \mathcal{L}\left(x, u_{\varepsilon}, \nabla u_{\varepsilon}\right) \cdot \nabla \psi_{\delta} d x \\
& +\int_{\Omega} D_{s} \mathcal{L}\left(x, u_{\varepsilon}, \nabla u_{\varepsilon}\right) u_{\varepsilon}\left(1-\psi_{\delta}\right) d x \\
& -\int_{\Omega}\left|u_{\varepsilon}\right|^{p^{*}-\varepsilon}\left(1-\psi_{\delta}\right) d x \\
\geq & \nu \int_{\Omega}\left|\nabla u_{\varepsilon}\right|^{p}\left(1-\psi_{\delta}\right) d x \\
& -\int_{\Omega} u_{\varepsilon} \nabla_{\xi} \mathcal{L}\left(x, u_{\varepsilon}, \nabla u_{\varepsilon}\right) \cdot \nabla \psi_{\delta} d x \\
& -\mathcal{L}^{n}(\Omega)^{\frac{\varepsilon}{p^{*}}}\left(\int_{\Omega}\left|u_{\varepsilon}\right|^{p^{*}}\left(1-\psi_{\delta}\right) d x\right)^{\frac{p^{*}-\varepsilon}{p^{*}}} .
\end{aligned}
$$

On the other hand, arguing as for (17), one obtains

$$
\lim _{\varepsilon \rightarrow 0}\left|\int_{\Omega} u_{\varepsilon} \nabla_{\xi} \mathcal{L}\left(x, u_{\varepsilon}, \nabla u_{\varepsilon}\right) \cdot \nabla \psi_{\delta} d x\right| \leq \beta_{\delta}
$$

for each $\delta>0$. Now, it results

$$
\begin{aligned}
\lim _{\varepsilon \rightarrow 0} \int_{\Omega}\left|\nabla u_{\varepsilon}\right|^{p}\left(1-\psi_{\delta}\right) d x & =\int_{\Omega}\left(1-\psi_{\delta}\right) d \mu \\
& \geq \int_{\Omega}|\nabla u|^{p}\left(1-\psi_{\delta}\right) d x+\sum_{j=1}^{r} \mu_{j}\left(1-\psi_{\delta}\left(x_{j}\right)\right) \\
& =\int_{\Omega}|\nabla u|^{p} d x+o(1)
\end{aligned}
$$


as $\delta \rightarrow 0$ and

$$
\begin{aligned}
\lim _{\varepsilon \rightarrow 0} \int_{\Omega}\left|u_{\varepsilon}\right|^{p^{*}}\left(1-\psi_{\delta}\right) d x & =\int_{\Omega}\left(1-\psi_{\delta}\right) d \sigma \\
& =\int_{\Omega}|u|^{p^{*}}\left(1-\psi_{\delta}\right) d x+\sum_{j=1}^{r} \sigma_{j}\left(1-\psi_{\delta}\left(x_{j}\right)\right) \\
& =\int_{\Omega}|u|^{p^{*}} d x+o(1)
\end{aligned}
$$

as $\delta \rightarrow 0$. Therefore, in view of (19) - (21), by letting $\delta \rightarrow 0$ and $\varepsilon \rightarrow 0$ in (18) one concludes that

$$
\nu \int_{\Omega}|\nabla u|^{p} d x \leq \int_{\Omega}|u|^{p^{*}} d x
$$

As $\Omega$ is bounded, by Proposition 3.1/(b) one has $\int_{\Omega}|\nabla u|^{p} d x>S\left(\int_{\Omega}|u|^{p^{*}} d x\right)^{\frac{p}{p^{*}}}$ which combined with (22) yields the assertion

Lemma 3.4. Let $\left(u_{\varepsilon}\right)_{\varepsilon>0} \subset W_{0}^{1, p}(\Omega)$ be a sequence of solutions of problem $\left(\mathcal{P}_{\varepsilon}\right)$ and let $u$ be its weak limit. Then $u$ is a solution of problem $\left(\mathcal{P}_{0}\right)$.

Proof. For each $\varepsilon>0$ and $\varphi \in C_{c}^{\infty}(\Omega)$,

$$
\int_{\Omega} \nabla_{\xi} \mathcal{L}\left(x, u_{\varepsilon}, \nabla u_{\varepsilon}\right) \cdot \nabla \varphi d x+\int_{\Omega} D_{s} \mathcal{L}\left(x, u_{\varepsilon}, \nabla u_{\varepsilon}\right) \varphi d x=\int_{\Omega}\left|u_{\varepsilon}\right|^{p^{*}-2-\varepsilon} u_{\varepsilon} \varphi d x .
$$

Since $\left(u_{\varepsilon}\right)_{\varepsilon>0}$ is bounded in $W_{0}^{1, p}(\Omega)$, up to a subsequence, $u$ satisfies

$$
\left.\begin{array}{clrl}
\nabla u_{\varepsilon} & \rightarrow \nabla u & & \text { in } L^{p}(\Omega) \\
u_{\varepsilon} & \rightarrow u & & \text { in } L^{p}(\Omega) \\
u_{\varepsilon}(x) & \rightarrow u(x) & & \text { for a.e. } x \in \Omega
\end{array}\right\}
$$

as $\varepsilon \rightarrow 0$. Moreover, by [7: Theorem 1], up to a further subsequence, we have $\nabla u_{\varepsilon}(x) \rightarrow \nabla u(x)$ for a.e. $x \in \Omega$. Therefore, in view of (5) one deduces that

$$
\nabla_{\xi} \mathcal{L}\left(x, u_{\varepsilon}, \nabla u_{\varepsilon}\right) \rightarrow \nabla_{\xi} \mathcal{L}(x, u, \nabla u) \quad \text { in } L^{p^{\prime}}\left(\Omega, \mathbb{R}^{n}\right) .
$$

By (4) - (6) one finds a constant $M>0$ such that for each $\delta>0$

$$
\left|D_{s} \mathcal{L}(x, s, \xi)\right| \leq M \nabla_{\xi} \mathcal{L}(x, s, \xi) \cdot \xi+a_{\delta}(x)+\delta|s|^{p^{*}}
$$

for a.e. $x \in \Omega$ and for all $(s, \xi) \in \mathbb{R} \times \mathbb{R}^{n}$. If we test equation (23) with the functions

$$
\varphi_{\varepsilon}=\varphi \exp \left\{-M u_{\varepsilon}^{+}\right\} \quad(\varepsilon>0)
$$


where $0 \leq \varphi \in W_{0}^{1, p} \cap L^{\infty}(\Omega)$, we obtain

$$
\begin{gathered}
\int_{\Omega} \nabla_{\xi} \mathcal{L}\left(x, u_{\varepsilon}, \nabla u_{\varepsilon}\right) \cdot \nabla \varphi \exp \left\{-M u_{\varepsilon}^{+}\right\} d x \\
-\int_{\Omega}\left|u_{\varepsilon}\right|^{p^{*}-2-\varepsilon} u_{\varepsilon} \varphi \exp \left\{-M u_{\varepsilon}^{+}\right\} d x \\
+\int_{\Omega}\left[D_{s} \mathcal{L}\left(x, u_{\varepsilon}, \nabla u_{\varepsilon}\right)-M \nabla_{\xi} \mathcal{L}\left(x, u_{\varepsilon}, \nabla u_{\varepsilon}\right) \cdot \nabla u_{\varepsilon}^{+}\right] \varphi \exp \left\{-M u_{\varepsilon}^{+}\right\} d x=0 .
\end{gathered}
$$

Since by inequalities (7) and (25) for each $\varepsilon>0$ and $\delta>0$ we have

$$
\left[D_{s} \mathcal{L}\left(x, u_{\varepsilon}, \nabla u_{\varepsilon}\right)-M \nabla_{\xi} \mathcal{L}\left(x, u_{\varepsilon}, \nabla u_{\varepsilon}\right) \cdot \nabla u_{\varepsilon}^{+}\right] \varphi \exp \left\{-M u_{\varepsilon}^{+}\right\}-\delta\left|u_{\varepsilon}\right|^{p^{*}} \leq a_{\delta}(x),
$$

arguing as in [18: Theorem 3.4] one obtains

$$
\begin{gathered}
\limsup _{\varepsilon \rightarrow 0} \int_{\Omega}\left[D_{s} \mathcal{L}\left(x, u_{\varepsilon}, \nabla u_{\varepsilon}\right)-M \nabla_{\xi} \mathcal{L}\left(x, u_{\varepsilon}, \nabla u_{\varepsilon}\right) \cdot \nabla u_{\varepsilon}^{+}\right] \varphi \exp \left\{-M u_{\varepsilon}^{+}\right\} d x \\
\leq \int_{\Omega}\left[D_{s} \mathcal{L}(x, u, \nabla u)-M \nabla_{\xi} \mathcal{L}(x, u, \nabla u) \cdot \nabla u^{+}\right] \varphi \exp \left\{-M u^{+}\right\} d x .
\end{gathered}
$$

Therefore, taking into account $(24)$ and since as $\varepsilon \rightarrow 0$

$$
\int_{\Omega}\left|u_{\varepsilon}\right|^{p^{*}-2-\varepsilon} u_{\varepsilon} \varphi d x \rightarrow \int_{\Omega}|u|^{p^{*}-2} u \varphi d x
$$

for each $0 \leq \varphi \in W_{0}^{1, p} \cap L^{\infty}(\Omega)$, one may conclude that

$$
\begin{gathered}
\int_{\Omega} \nabla_{\xi} \mathcal{L}(x, u, \nabla u) \cdot \nabla \varphi \exp \left\{-M u^{+}\right\} d x \\
-\int_{\Omega}|u|^{p^{*}-2} u \varphi \exp \left\{-M u^{+}\right\} d x \\
+\int_{\Omega}\left[D_{s} \mathcal{L}(x, u, \nabla u)-M \nabla_{\xi} \mathcal{L}(x, u, \nabla u) \cdot \nabla u^{+}\right] \varphi \exp \left\{-M u^{+}\right\} d x \geq 0
\end{gathered}
$$

for each $0 \leq \varphi \in W_{0}^{1, p} \cap L^{\infty}(\Omega)$. Testing now (26) with $\varphi_{k}=\varphi \vartheta\left(\frac{u}{k}\right) \exp \left\{M u^{+}\right\}$ where $0 \leq \varphi \in C_{c}^{\infty}(\Omega)$ and $\vartheta$ is smooth, $\vartheta=1$ in $\left[-\frac{1}{2}, \frac{1}{2}\right]$ and $\vartheta=0$ in $(-\infty,-1] \cup[1,+\infty)$, it follows that

$$
\begin{gathered}
\int_{\Omega} \nabla_{\xi} \mathcal{L}(x, u, \nabla u) \cdot \nabla \varphi_{k} \exp \left\{-M u^{+}\right\} d x \\
-\int_{\Omega}|u|^{p^{*}-2} u \varphi \vartheta\left(\frac{u}{k}\right) d x \\
+\int_{\Omega}\left[D_{s} \mathcal{L}(x, u, \nabla u)-M \nabla_{\xi} \mathcal{L}(x, u, \nabla u) \cdot \nabla u^{+}\right] \varphi \vartheta\left(\frac{u}{k}\right) d x \geq 0
\end{gathered}
$$


which, arguing again as in [18: Theorem 3.4], yields as $k \rightarrow+\infty$

$$
\int_{\Omega} \nabla_{\xi} \mathcal{L}(x, u, \nabla u) \cdot \nabla \varphi d x+\int_{\Omega} D_{s} \mathcal{L}(x, u, \nabla u) \varphi d x \geq \int_{\Omega}|u|^{p^{*}-2} u \varphi d x
$$

for each $0 \leq \varphi \in C_{c}^{\infty}(\Omega)$. Analogously, testing with $\varphi_{\varepsilon}=\varphi \exp \left\{-M u_{\varepsilon}^{-}\right\}$, one obtains the opposite inequality, i.e. $u$ is a solution of problem $\left(\mathcal{P}_{0}\right)$

\section{Proofs of the main results}

Let now $\left(u_{\varepsilon}\right)_{\varepsilon>0}$ be a sequence of solutions of problem $\left(\mathcal{P}_{\varepsilon}\right)$ with $f_{\varepsilon}\left(u_{\varepsilon}\right) \rightarrow c$ and

$$
\frac{p^{*}-p-\gamma}{p p^{*}}(\nu S)^{\frac{n}{p}}<c<2 \frac{p^{*}-p-\gamma}{p p^{*}}(\nu S)^{\frac{n}{p}} .
$$

Then there exist a subsequence of $\left(u_{\varepsilon}\right)_{\varepsilon>0}$ and two bounded positive measures $\mu$ and $\sigma$ verifying (10) - (13).

Proof of Theorem 2.7. Let us first show that there exists at most one $j$ such that $\mu_{j} \neq 0$. Suppose that $\mu_{j} \neq 0$ for $j=1, \ldots, r$; in view of Lemma 3.2 one has $\mu_{j} \geq \nu^{\frac{n}{p^{*}}} S^{\frac{n}{p}}$. Following the proof of Lemma 2.5, we obtain

$$
\begin{aligned}
c & =\lim _{\varepsilon \rightarrow 0} f_{\varepsilon}\left(u_{\varepsilon}\right) \\
& \geq \frac{p^{*}-p-\gamma}{p p^{*}} \nu \lim _{\varepsilon \rightarrow 0} \int_{\Omega}\left|\nabla u_{\varepsilon}\right|^{p} d x \\
& \geq \frac{p^{*}-p-\gamma}{p p^{*}} \nu \int_{\Omega} d \mu \\
& \geq \frac{p^{*}-p-\gamma}{p p^{*}} \nu \sum_{j=1}^{r} \mu_{j} \\
& \geq r \frac{p^{*}-p-\gamma}{p p^{*}}(\nu S)^{\frac{n}{p}} .
\end{aligned}
$$

Taking into account (27) one has

$$
2 \frac{p^{*}-p-\gamma}{p p^{*}}(\nu S)^{\frac{n}{p}}>c \geq r \frac{p^{*}-p-\gamma}{p p^{*}}(\nu S)^{\frac{n}{p}}
$$

hence $r \leq 1$. Now, arguing again as in Lemma 2.5 one obtains

$$
\begin{aligned}
2 \frac{p^{*}-p-\gamma}{p p^{*}}(\nu S)^{\frac{n}{p}}>c & =\lim _{\varepsilon \rightarrow 0} f_{\varepsilon}\left(u_{\varepsilon}\right) \\
& \geq \frac{p^{*}-p-\gamma}{p p^{*}} \nu \lim _{\varepsilon \rightarrow 0} \int_{\Omega}\left|\nabla u_{\varepsilon}\right|^{p} d x \\
& \geq \frac{p^{*}-p-\gamma}{p p^{*}}\left(\nu \int_{\Omega}|\nabla u|^{p} d x+\nu \mu_{1}\right) .
\end{aligned}
$$


If both summands were non-zero, by Lemmas 3.2 and 3.3 we would obtain

$$
\begin{aligned}
\nu \int_{\Omega}|\nabla u|^{p} d x & >(\nu S)^{\frac{n}{p}} \\
\nu \mu_{1} & \geq(\nu S)^{\frac{n}{p}}
\end{aligned}
$$

and thus a contradiction. Vice versa, let us assume that $u=0$ and $\mu_{1}=0$. Let $0 \leq \psi \in C_{c}^{1}(\Omega)$. By testing our equation with $\psi u_{\varepsilon}$ and using Hölder inequality, one gets

$$
\begin{aligned}
\int_{\Omega} u_{\varepsilon} \nabla_{\xi} \mathcal{L}\left(x, u_{\varepsilon}, \nabla u_{\varepsilon}\right) \cdot \nabla \psi d x & \\
+\int_{\Omega} \psi \nabla_{\xi} \mathcal{L}\left(x, u_{\varepsilon}, \nabla u_{\varepsilon}\right) \cdot \nabla u_{\varepsilon} d x & \\
+\int_{\Omega} D_{s} \mathcal{L}\left(x, u_{\varepsilon}, \nabla u_{\varepsilon}\right) \psi u_{\varepsilon} d x & =\int_{\Omega}\left|u_{\varepsilon}\right|^{p^{*}-\varepsilon} \psi d x \\
\leq & \left(\int_{\Omega}\left|u_{\varepsilon}\right|^{p^{*}} \psi d x\right)^{\frac{p^{*}-\varepsilon}{p^{*}}} \mathcal{L}^{n}(\Omega)^{\frac{\varepsilon}{p^{*}}}
\end{aligned}
$$

Since $\left(u_{\varepsilon}\right)_{\varepsilon>0}$ is bounded in $W_{0}^{1, p}(\Omega)$, by (5) there exists a constant $C>0$ such that

$$
\left|\int_{\Omega} u_{\varepsilon} \nabla_{\xi} \mathcal{L}\left(x, u_{\varepsilon}, \nabla u_{\varepsilon}\right) \cdot \nabla \psi d x\right| \leq C\left\|u_{\varepsilon}\right\|_{p}
$$

which by $u_{\varepsilon} \rightarrow 0$ in $L^{p}(\Omega)$ yields

$$
\lim _{\varepsilon \rightarrow 0} \int_{\Omega} u_{\varepsilon} \nabla_{\xi} \mathcal{L}\left(x, u_{\varepsilon}, \nabla u_{\varepsilon}\right) \cdot \nabla \psi d x=0 .
$$

Moreover, since by (7) we get

$$
\int_{\Omega} D_{s} \mathcal{L}\left(x, u_{\varepsilon}, \nabla u_{\varepsilon}\right) \psi u_{\varepsilon} d x \geq 0
$$

taking into account (6) and passing to the limit in (28) we get

$$
\forall \psi \in C_{c}(\Omega): \quad \psi \geq 0 \quad \Longrightarrow \quad \nu \int_{\Omega} \psi d \mu \leq \int_{\Omega} \psi d \sigma .
$$

On the other hand, $\mu_{1}=0$ and $u=0$ imply $\sigma=0$. Then, since $\mu \geq 0$, by 
(29) we get $\mu=0$. In particular, by (3), (6) and (7) one gets

$$
\begin{aligned}
c= & \lim _{\varepsilon \rightarrow 0} f_{\varepsilon}\left(u_{\varepsilon}\right) \\
= & \lim _{\varepsilon \rightarrow 0}\left[\int_{\Omega} \mathcal{L}\left(x, u_{\varepsilon}, \nabla u_{\varepsilon}\right) d x\right. \\
& -\frac{1}{p^{*}-\varepsilon} \int_{\Omega} \nabla_{\xi} \mathcal{L}\left(x, u_{\varepsilon}, \nabla u_{\varepsilon}\right) \cdot \nabla u_{\varepsilon} d x \\
& \left.-\frac{1}{p^{*}-\varepsilon} \int_{\Omega} D_{s} \mathcal{L}\left(x, u_{\varepsilon}, \nabla u_{\varepsilon}\right) u_{\varepsilon} d x\right] \\
\leq & b_{0} \lim _{\varepsilon \rightarrow 0}\left(\int_{\Omega}\left|u_{\varepsilon}\right|^{p} d x+\int_{\Omega}\left|\nabla u_{\varepsilon}\right|^{p} d x\right) \\
= & b_{0} \int_{\Omega} d \mu \\
= & 0,
\end{aligned}
$$

which is not possible. Therefore, either $\mu_{1}=0$ and $u \neq 0$, or $\mu_{1} \neq 0$ and $u=0$

Remark 4.1. If $(27)$ is replaced by the $(k+1)$-th critical energy range

$$
k \frac{p^{*}-p-\gamma}{p p^{*}}(\nu S)^{\frac{n}{p}}<c<(k+1) \frac{p^{*}-p-\gamma}{p p^{*}}(\nu S)^{\frac{n}{p}}
$$

for $k \in \mathbb{N}$, in a similar way one proves that $\mu_{j}=0$ for any $j \geq k+1$ and there holds:

(a) If $\mu_{j}=0$ for every $j \geq 1$, then $u$ is a non-trivial solution of problem $\left(\mathcal{P}_{0}\right)$.

(b) If $\mu_{j} \neq 0$ for every $1 \leq j \leq k$, then $u=0$.

Remark 4.2. Let $f_{0}: W_{0}^{1, p}(\Omega) \rightarrow \mathbb{R}$ be the functional associated with problem $\left(\mathcal{P}_{0}\right)$ and let $0 \neq u \in W_{0}^{1, p}(\Omega)$ be a solution of problem $\left(\mathcal{P}_{0}\right)$ (obtained as weak limit of $\left.\left(u_{\varepsilon}\right)_{\varepsilon>0}\right)$. Then

$$
f_{0}(u)>\frac{p^{*}-p-\gamma}{p p^{*}}(\nu S)^{\frac{n}{p}}
$$

Indeed,

$$
\begin{aligned}
f_{0}(u) & =f_{0}(u)-\frac{1}{p^{*}} f_{0}^{\prime}(u)(u) \\
& \geq \frac{p^{*}-p-\gamma}{p^{*}} \int_{\Omega} \mathcal{L}(x, u, \nabla u) d x \\
& \geq \frac{p^{*}-p-\gamma}{p p^{*}} \nu \int_{\Omega}|\nabla u|^{p} d x
\end{aligned}
$$


which yields (30) in view of Lemma 3.3. This, in some sense, explains why one chooses $c$ greater than $\frac{p^{*}-p-\gamma}{p p^{*}}(\nu S)^{\frac{n}{p}}$ in Theorem 2.7.

Let now $\left(u_{\varepsilon}\right)_{\varepsilon>0}$ be a sequence of solutions of problem $\left(\mathcal{P}_{\varepsilon}\right)$ with $f_{\varepsilon}\left(u_{\varepsilon}\right) \rightarrow$ $c$ and

$$
\lim _{\varepsilon \rightarrow 0} f_{\varepsilon}\left(u_{\varepsilon}\right)=\frac{p^{*}-p-\gamma}{p p^{*}}(\nu S)^{\frac{n}{p}}
$$

Proof of Theorem 2.8. Let us first note that

$$
f_{0}(u) \leq \lim _{\varepsilon \rightarrow 0} f_{\varepsilon}\left(u_{\varepsilon}\right)+\frac{1}{p^{*}} \sum_{j=1}^{\infty} \sigma_{j}
$$

Indeed, taking into account that by [6: Theorem 3.4]

$$
\int_{\Omega} \mathcal{L}(x, u, \nabla u) d x \leq \lim _{\varepsilon \rightarrow 0} \int_{\Omega} \mathcal{L}\left(x, u_{\varepsilon}, \nabla u_{\varepsilon}\right) d x
$$

(31) follows by combining Hölder inequality with (12).

Now assume by contradiction that $u \neq 0$. Then there exists $j_{0} \in \mathbb{N}$ such that $\mu_{j_{0}} \neq 0$ and $\sigma_{j_{0}} \neq 0$, otherwise by Remark 4.2 and (31) we would get

$$
\frac{p^{*}-p-\gamma}{p p^{*}}(\nu S)^{\frac{n}{p}}<f_{0}(u) \leq \lim _{\varepsilon \rightarrow 0} f_{\varepsilon}\left(u_{\varepsilon}\right)=\frac{p^{*}-p-\gamma}{p p^{*}}(\nu S)^{\frac{n}{p}}
$$

Arguing as in Lemma 2.5 and applying Lemma 3.2, we obtain

$$
\begin{aligned}
\frac{p^{*}-p-\gamma}{p p^{*}}(\nu S)^{\frac{n}{p}} & =\lim _{\varepsilon \rightarrow 0} f_{\varepsilon}\left(u_{\varepsilon}\right) \\
& \geq \frac{p^{*}-p-\gamma}{p p^{*}}\left(\nu \int_{\Omega}|\nabla u|^{p} d x+\nu \mu_{j_{0}}\right) \\
& \geq \frac{p^{*}-p-\gamma}{p p^{*}} \nu \int_{\Omega}|\nabla u|^{p} d x+\frac{p^{*}-p-\gamma}{p p^{*}}(\nu S)^{\frac{n}{p}}
\end{aligned}
$$

which implies $u=0-$ a contradiction 


\section{Mountain-pass critical values}

In this section, we shall investigate the asymptotics of $\left(u_{\varepsilon}\right)$ in the case of critical levels of min-max type. We assume that $\mathcal{L}$ is $p$-homogeneous with respect to $\xi$ and satisfies a stronger assumption, i.e.

$$
\mathcal{L}(x, s, \xi) \leq \frac{1}{p}|\xi|^{p}
$$

for a.e. $x \in \Omega$ and for all $(s, \xi) \in \mathbb{R} \times \mathbb{R}^{n}$. In particular, it results that $\nu \leq 1$. Let $u_{\varepsilon}$ be a critical point of $f_{\varepsilon}$ associated with the mountain pass level

$$
c_{\varepsilon}=\inf _{\eta \in \mathcal{C}_{\varepsilon}} \max _{t \in[0,1]} f_{\varepsilon}(\eta(t))
$$

where

$$
\mathcal{C}_{\varepsilon}=\left\{\eta \in C\left([0,1], W_{0}^{1, p}(\Omega)\right): \eta(0)=0 \text { and } \eta(1)=w_{\varepsilon}\right\}
$$

and $w_{\varepsilon} \in W_{0}^{1, p}(\Omega)$ is chosen in such a way that $f_{\varepsilon}\left(w_{\varepsilon}\right)<0$.

Lemma 5.1. The inequality $\lim _{\varepsilon \rightarrow 0} f_{\varepsilon}\left(u_{\varepsilon}\right) \leq \frac{1}{n} S^{\frac{n}{p}}$ holds.

Proof. Let $x_{0} \in \Omega$ and $\delta>0$, and consider the functions $T_{\delta, x_{0}}$ as in (15). By Proposition 3.1/(c) one has

$$
\left\|\nabla T_{\delta, x_{0}}\right\|_{p, \mathbb{R}^{n}}^{p}=\left\|T_{\delta, x_{0}}\right\|_{p^{*}, \mathbb{R}^{n}}^{p^{*}}=S^{\frac{n}{p}} .
$$

Moreover, taking a function $\phi \in C_{c}^{\infty}(\Omega)$ with $0 \leq \phi \leq 1$ and $\phi=1$ in a neighbourhood of $x_{0}$ and setting $v_{\delta}=\phi T_{\delta, x_{0}}$, it results

$$
\left.\begin{array}{rl}
\left\|\nabla v_{\delta}\right\|_{p}^{p} & =S^{\frac{n}{p}}+o(1) \\
\left\|v_{\delta}\right\|_{p^{*}}^{p^{*}} & =S^{\frac{n}{p}}+o(1)
\end{array}\right\} \quad(\delta \rightarrow 0)
$$

(see [10: Lemma 3.2]).

We want to prove that, for any $t \geq 0$,

$$
\lim _{\varepsilon \rightarrow 0} f_{\varepsilon}\left(t v_{\delta}\right) \leq \frac{1}{n} S^{\frac{n}{p}}+o(1) \quad(\delta \rightarrow 0) .
$$

By (32) one has

$$
\begin{aligned}
\lim _{\varepsilon \rightarrow 0} f_{\varepsilon}\left(t v_{\delta}\right) & =t^{p} \int_{\Omega} \mathcal{L}\left(x, t v_{\delta}, \nabla v_{\delta}\right) d x-\lim _{\varepsilon \rightarrow 0} \frac{t^{p^{*}-\varepsilon}}{p^{*}-\varepsilon} \int_{\Omega}\left|v_{\delta}\right|^{p^{*}-\varepsilon} d x \\
& \leq \frac{t^{p}}{p} \int_{\Omega}\left|\nabla v_{\delta}\right|^{p} d x-\frac{t^{p^{*}}}{p^{*}} \int_{\Omega}\left|v_{\delta}\right|^{p^{*}} d x
\end{aligned}
$$

Keeping into account (34) and the fact that $\frac{t^{p}}{p}-\frac{t^{p^{*}}}{p^{*}} \leq \frac{1}{n}$ for every $t \geq 0$, one gets

$$
\lim _{\varepsilon \rightarrow 0} f_{\varepsilon}\left(t v_{\delta}\right) \leq \frac{t^{p}}{p} S^{\frac{n}{p}}-\frac{t^{p^{*}}}{p^{*}} S^{\frac{n}{p}}+o(1) \leq \frac{1}{n} S^{\frac{n}{p}}+o(1) \quad(\delta \rightarrow 0) .
$$

Now choose $t_{0}>0$ such that $f_{\varepsilon}\left(t_{0} v_{\delta}\right)<0$; by (33) we have

$$
\lim _{\varepsilon \rightarrow 0} f_{\varepsilon}\left(u_{\varepsilon}\right) \leq \lim _{\varepsilon \rightarrow 0} \max _{s \in[0,1]} f_{\varepsilon}\left(s t_{0} v_{\delta}\right) \leq \frac{1}{n} S^{\frac{n}{p}}+o(1)
$$

and this, by letting $\delta \rightarrow 0$, ends up the proof 
Theorem 5.2. Suppose that the number of non-zero Dirac masses is

$$
\left[\frac{p p^{*}}{\left(p^{*}-p-\gamma\right) n \nu^{\frac{n}{p}}}\right]
$$

where $[x]$ denotes the integer part of $x$. Then $u=0$.

Proof. Keeping into account the previous lemma and arguing as in Lemmal 2.5

$$
\begin{aligned}
\frac{1}{n} S^{\frac{n}{p}} & \geq \lim _{\varepsilon \rightarrow 0} f_{\varepsilon}\left(u_{\varepsilon}\right) \\
& \geq \frac{p^{*}-p-\gamma}{p p^{*}} \nu\left(\int_{\Omega}|\nabla u|^{p} d x+\sum_{j=1}^{r} \mu_{j}\right) \\
& \geq \frac{p^{*}-p-\gamma}{p p^{*}} \nu \int_{\Omega}|\nabla u|^{p} d x+r \frac{p^{*}-p-\gamma}{p p^{*}} \nu^{\frac{n}{p}} S^{\frac{n}{p}}
\end{aligned}
$$

where $r$ denotes the number of non-vanishing masses. Hence it must be

$$
0 \leq r \leq\left[\frac{p p^{*}}{\left(p^{*}-p-\gamma\right) n \nu^{\frac{n}{p}}}\right]
$$

In particular, if $r$ is maximum and $u \neq 0$, by virtue of Lemma 3.3 one obtains

$$
\frac{p^{*}-p-\gamma}{p p^{*}} \nu^{\frac{n}{p}} S^{\frac{n}{p}}>\frac{p^{*}-p-\gamma}{p p^{*}} \nu \int_{\Omega}|\nabla u|^{p} d x>\frac{p^{*}-p-\gamma}{p p^{*}} \nu^{\frac{n}{p}} S^{\frac{n}{p}}
$$

which is a contradiction

\section{Final remarks}

Assume that $\mathcal{L}(x, s, \xi)$ is of class $C^{1}$ in $\bar{\Omega} \times \mathbb{R} \times \mathbb{R}^{n}$ and, additionally, that the vector-valued function

$$
\nabla_{\xi} \mathcal{L}(x, s, \xi)=\left(\frac{\partial \mathcal{L}}{\partial \xi_{1}}(x, s, \xi), \ldots, \frac{\partial \mathcal{L}}{\partial \xi_{n}}(x, s, \xi)\right)
$$

is of class $C^{1}$ in $\bar{\Omega} \times \mathbb{R} \times \mathbb{R}^{n}$.

Theorem 6.1. Let $\Omega$ be star-shaped with respect to the origin and assume that

$$
p^{*} \nabla_{x} \mathcal{L}(x, s, \xi) \cdot x-n D_{s} \mathcal{L}(x, s, \xi) s \geq 0
$$

for $(x, s, \xi) \in \Omega \times \mathbb{R} \times \mathbb{R}^{n}$. Then $\left(\mathcal{P}_{0}\right)$ has no non-trivial solution u in $C^{2}(\Omega) \cap$ $C^{1}(\bar{\Omega})$. 
Proof. Let $\widehat{\mathcal{L}}: \Omega \times \mathbb{R} \times \mathbb{R}^{n} \rightarrow \mathbb{R}$ by defined by setting

$$
\widehat{\mathcal{L}}(x, s, \xi)=\mathcal{L}(x, s, \xi)-\frac{1}{p^{*}}|s|^{p^{*}}
$$

for all $(x, s, \xi) \in \Omega \times \mathbb{R} \times \mathbb{R}^{n}$. Then apply the Pucci-Serrin inequality [17]

$$
n \widehat{\mathcal{L}}+\nabla_{x} \widehat{\mathcal{L}} \cdot x-a D_{s} \widehat{\mathcal{L}} s-(a+1) \nabla_{\xi} \widehat{\mathcal{L}} \cdot \xi \geq 0
$$

with the choice $a=\frac{n-p}{p}$

Remark 6.2. If $\Omega$ is star-shaped and $\mathcal{L}$ does not depend on $x$, then problem $\left(\mathcal{P}_{0}\right)$ admits no non-trivial solution in $C^{2}(\Omega) \cap C^{1}(\bar{\Omega})$ when $D_{s} \mathcal{L}(s, \xi) s \leq 0$, which is the opposite of (7). In particular, (7) seems to be a natural assumption.

Remark 6.3. As noted in the introduction, if $\Omega$ is star-shaped and $\mathcal{L}(\xi)=|\xi|^{p} / p$, in [10] it is proven that problem $\left(\mathcal{P}_{0}\right)$ has no non-trivial solution in $W_{0}^{1, p}(\Omega)$. In particular, by Theorem 2.7 one has $\mu_{1} \neq 0$.

Acknowledgement. The authors wish to thank M. Degiovanni for providing some useful discussions.

\section{References}

[1] Arcoya, D. and L. Boccardo: Critical points for multiple integrals of the calculus of variations. Arch. Ration. Mech. Anal. 134 (1996), 249 - 274.

[2] Atkinson, F. V. and L. A. Peletier: Elliptic equations with nearly critical growth. J. Diff. Equ. 70 (1987), 349 - 365.

[3] Bahri, A. and J. M. Coron: On a nonlinear elliptic equation involving the critical Sobolev exponent: the effect of the topology of the domain. Comm. Pure Appl. Math. 41 (1988), 253 - 294.

[4] Bandle, C. and A. Brillard: Nonlinear elliptic equations involving critical Soboler exponents: Asymptotic analysis via methods of epi-convergence. Z. Anal. Anw. 13 (1994), $615-628$.

[5] Brézis, H. and F. E. Browder: Some properties of higher order Sobolev spaces. J. Math. Pures Appl. 61 (1982), 245 - 259.

[6] Dacorogna, B.: Direct Methods in the Calculus of Variations. Berlin: SpringerVerlag 1988.

[7] Dal Maso, G. and F. Murat: Almost everywhere convergence of the gradients of solutions to nonlinear elliptic systems. Nonlin. Anal. 31 (1998), $405-412$.

[8] Dancer, E. N.: A note on an equation with critical exponent. Bull. London Math. Soc. 20 (1988), $600-602$. 
[9] Garcia Azorero, J. and I. Peral Alonso: On limits of solutions of elliptic problems with nearly critical exponent. Comm. Part. Diff. Equ. 17 (1992), 2113 2126.

[10] Guedda, M. and L. Veron: Quasilinear elliptic problems involving critical Sobolev exponents. Nonlin. Anal. 13 (1989), 879 - 902.

[11] Kazdan, J. L. and F. W. Warner: Remarks on some quasilinear elliptic equations. Comm. Pure Appl. Math. 28 (1975), 567 - 597.

[12] Knaap, M. C. and L. A. Peletier: Quasilinear elliptic equations with nearly critical growth. Comm. Part. Diff. Equ. 14 (1989), $1351-1383$.

[13] Lions, P. L.: The concentration-compactness principle in the calculus of variations. Part I: The limit case. Rev. Mat. Iberoamer. 1 (1985), (1) $145-$ 201.

[14] Lions, P. L.: The concentration-compactness principle in the calculus of variations. Part II: The limit case. Rev. Mat. Iberoamer. 1 (1985), (2) 45 121.

[15] Passaseo, D.: The effect of the domain shape on the existence of positive solutions of the equation $\Delta u+u^{2^{*}-1}=0$. Top. Meth. Nonlin. Anal. 3 (1994), 27 -54 .

[16] Pellacci, B.: Critical points for non-differentiable functionals. Bull. Un. Mat. Ital. B (7) 11 (1997), $733-749$.

[17] Pucci, P. and J. Serrin: A general variational identity. Indiana Univ. Math. J. 35 (1986), $681-703$.

[18] Squassina, M.: Existence of weak solutions to general Euler's equations via nonsmooth critical point theory. Ann. Fac. Sci. Toulouse Math. (6) 9 (2000), $113-131$.

[19] Talenti, G.: Best constant in Sobolev inequality. Ann. Mat. Pura Appl. (4) 110 (1976), $353-372$.

Received 07.06.2001 